\title{
Technè
}

La science au service de l'histoire de l'art et de la préservation des biens culturels

47 | 2019

Bernard Palissy : nouveaux regards sur la céramique française auX $\mathrm{XVI}{ }^{\mathrm{e}}$ et $\mathrm{XVII}{ }^{\mathrm{e}}$ siècles

\section{Les grands moules en plâtre du fonds Palissy conservés au musée national de la Renaissance, Écouen}

Large plaster moulds in the Palissy collection now in the Musée national de la Renaissance, Écouen

\section{Anne Courcelle et Véronique Picur}

\section{OpenEdition Journals}

Édition électronique

URL : http://journals.openedition.org/techne/1449

DOI : $10.4000 /$ techne.1449

ISSN : 2534-5168

Éditeur

C2RMF

Édition imprimée

Date de publication : 1 juin 2019

Pagination : $33-41$

ISBN : 978-2-11-152830-7

ISSN : $1254-7867$

Référence électronique

Anne Courcelle et Véronique Picur, «Les grands moules en plâtre du fonds Palissy conservés au musée national de la Renaissance, Écouen », Technè [En ligne], 47 | 2019, mis en ligne le 01 juin 2020, consulté le 22 juillet 2020. URL : http://journals.openedition.org/techne/1449 ; DOI : https://doi.org/ 10.4000/techne.1449

\section{(c) (i) (2)}

La revue Technè. La science au service de l'histoire de l'art et de la préservation des biens culturels est mise à disposition selon les termes de la Licence Creative Commons Attribution - Pas d'Utilisation Commerciale - Pas de Modification 4.0 International. 
Anne Courcelle

Véronique Picur

\section{Les grands moules en plâtre du fonds Palissy conservés au musée national de la Renaissance, Écouen}

Large plaster moulds in the Palissy collection now in the Musée national de la Renaissance, Écouen

Résumé. À la suite des deux campagnes de fouilles menées entre 1983 et 1990 dans le jardin des Tuileries, de nombreux fragments de moules ont été découverts, dont une série reconstituant des grands moules de personnages féminins et masculins drapés à l'antique ou habillés d'éléments de la nature qui devaient orner les grottes conçues par Bernard Palissy. Notre intervention poursuit celle des archéologues. Une quarantaine de moules ont été identifiés dont quinze portraits, ce qui supposerait autant de personnages, antiques et termes.

Les contraintes techniques liées à l'état de conservation des moules et à leur nature même (représentation en "négatif ») ne permet pas de proposer d'assemblages certains. La poursuite des travaux par remontage des moules encore à l'état de fragments pourrait rendre possible la prise de leurs dimensions internes et permettrait une lecture globale en lumière rasante qui faciliterait la lecture des modelés. L'étude en cours des moules des décors architecturaux complètera les informations que ce matériel, conservé au château d'Écouen, apporte à la compréhension des grottes palisséennes.

Mots-clés. Palissy, grotte, moule à creux perdu, moulage, terme, terme métamorphosé, antique, coquillages, cailloux, nature, terre, argile, plâtre, décor rustique, Tuileries, rocaille.
Abstract. Following the excavations conducted between 1983 and 1990 in the Tuileries gardens, numerous fragments of moulds were discovered, including a series forming large moulds of male and female figures in classical drapery or clad in natural elements that must have adorned the grottoes designed by Bernard Palissy. Our activities commenced when the archaeologists had completed their operations. Forty or so moulds were identified, including fifteen portraits, which would suggest a similar number of figures, antiques and terms. The technical constraints relating to the condition of the moulds and their very nature (negative images) do not allow us to propose infallible assemblages. Pursuing our research by reassembling moulds still in fragments could make it possible to take their internal measurements and to examine them globally in a raking light that would facilitate our reading of the modelling. Current research on moulds used in architectural ornamentation will complement the information that this material, now in the château d'Écouen, adds to our understanding of Palissian grottoes.

Keywords. Palissy, grotto, waste mould, casting, term, metamorphic term, antique, shells, pebbles, nature, earth, clay, plaster, rusticware, Tuileries, rocaille.
En 1555-1556, le connétable Anne de Montmorency passa commande à Bernard Palissy d'une grotte en terre cuite glaçurée plombifère pour l'un de ses châteaux. Les aléas religieux et politiques empêchèrent ce dernier de la construire. L'ensemble décrit dans son ouvrage Architecture et ordonnance de la grotte rustique, en 1563, concerne peut-être cette grotte.

Catherine de Médicis lui commanda en 1565 une grotte pour le Palais des Tuileries, sur le modèle de celle qu'il était en train de faire pour le Connétable. Certains de ces éléments pourraient avoir été intégrés à la grotte des Tuileries. Vraisemblablement édifiée en partie, elle fut détruite ou tomba rapidement en ruine ${ }^{1}$.

Au cours du percement d'une tranchée dans la cour du Carrousel au Palais des Tuileries, en 1878, on découvrit plus d'une soixantaine de fragments supposés provenir de l'atelier parisien de Bernard Palissy. Ces fragments étaient les premiers témoins des travaux de Palissy concernant la grotte, jusque-là connue uniquement par des écrits et des archives. Entre 1983 et 1990, les fouilles du jardin des Tuileries (voir l'article de A. Gerbier dans ce volume) confirmèrent l'existence d'ateliers de céramique de tuiliers dans la cour du Carrousel, dont celui utilisé par Bernard Palissy pour modeler et cuire les divers éléments de la grotte des Tuileries.

Les fouilles ont livré un important corpus palisséen composé de plusieurs ensembles : les petits et grands moules en plâtre, les moules en terre cuite, les tirages glaçurés ou non, les éléments de vaisselle, les carreaux et de nombreux fragments liés aux techniques de fabrication. 


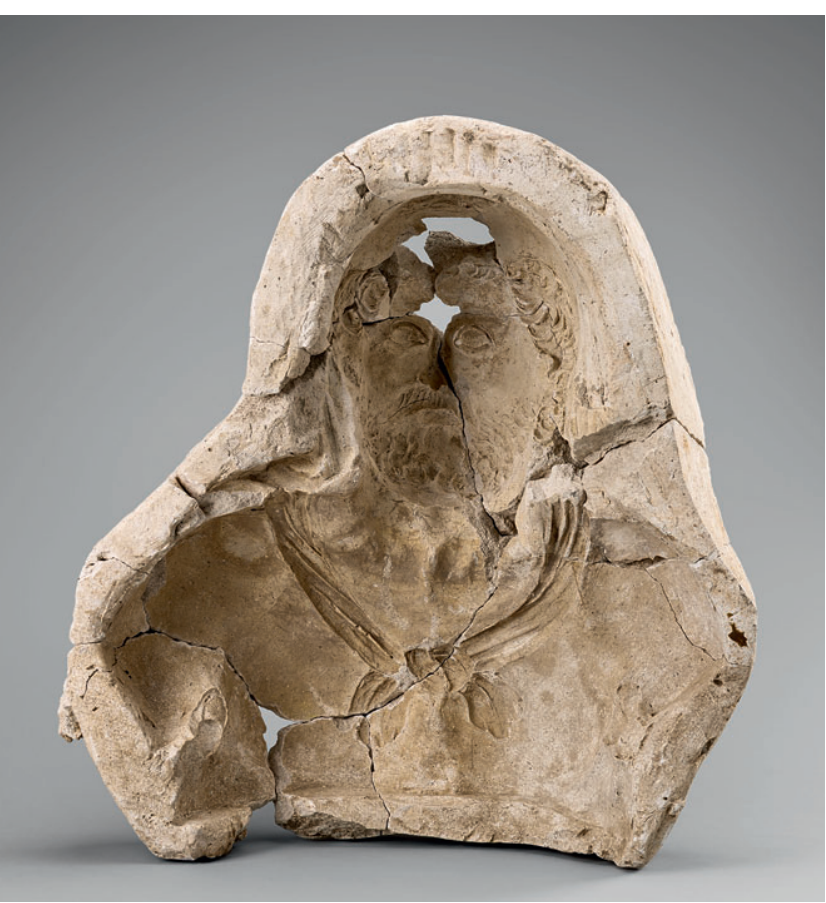

Fig. 1 a. Atelier de Bernard Palissy, Paris, fouilles des Tuileries, troisième quart du Xvi ${ }^{\mathrm{e}}$ siècle, Moule d'un buste d'homme portant une léonté (Hercule ?), plâtre (H. 60,5 cm, L. $55 \mathrm{~cm}, \operatorname{Pr} .23 \mathrm{~cm}$ ), Écouen, musée national de la Renaissance, EP 2717.

(c) RMN-Grand Palais (musée national de la Renaissance, château d'Écouen)/Mathieu Rabeau.

Cet article propose un premier bilan de nos travaux sur les grands moules en plâtre, destinés à l'une ou l'autre grotte, retrouvés, d'après les archéologues ${ }^{2}$, dans une petite annexe latérale d'une partie de l'ancien four de tuilier, rangés et empilés avec soin.

Pour la plupart cassés en de nombreux fragments, ces moules ont déjà fait l'objet d'une première campagne de recherche de collages par les archéologues au cours de la fouille. Un certain nombre d'entre eux ont ainsi été reconstitués, parfois partiellement. Nous avons repris ces travaux dans les réserves du musée national de la Renaissance au château d'Écouen et, après plusieurs années de recherche de collages et d'observations techniques, une quarantaine de moules ont été identifiés. Certains sont composés de nombreux fragments : par exemple, 26 morceaux ont été comptabilisés jusqu'à présent pour le moule inventorié EP 2713, encore incomplet. La plupart des moules restent lacunaires.

Le matériel étudié représente des personnages féminins et masculins de différents types, drapés à l'antique ou habillés d'éléments de la nature.

Pour mener à bien nos travaux, nous avons croisé plusieurs sources : bibliographiques, techniques et analytiques. À la recherche de collages étaient associés des examens en lumière rasante pour la lecture des marques techniques (traces d'outils, empreinte de matériaux organiques), sous loupe binoculaire afin de caractériser le plâtre et ses adjuvants (présence ou non de minéraux, de charbon), les dépôts présents en surface (sédiments liés à l'enfouissement ou

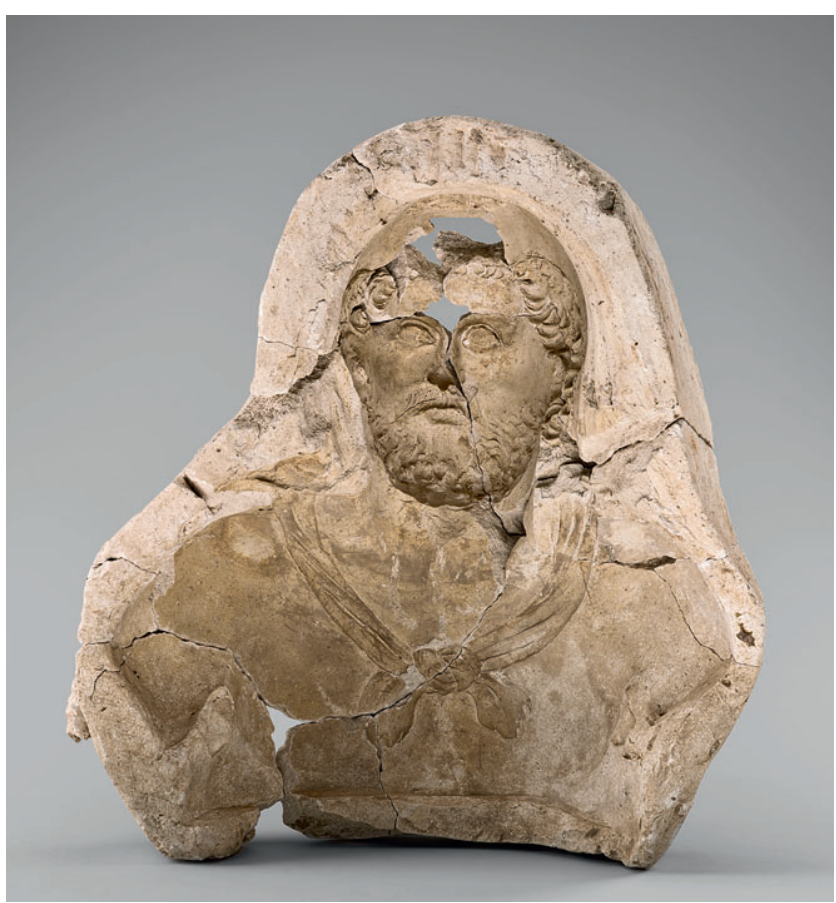

Fig. 1 b. Prise de vue en lumière rasante donnant l'effet en positif du moule 1 a. (C) RMN-Grand Palais (musée national de la Renaissance, château d'Écouen)/Mathieu Rabeau.

restes d'argile), et de rechercher des agents démoulants. Des prélèvements d'argile et de plâtre ont été confiés au C2RMF.

\section{La typologie des grands moules}

Les moules observés représentent des personnages qui peuvent être classés en trois catégories : des figures humaines à l'antique, des termes métamorphosés dont les visages et les différentes parties du corps sont recouverts de coquillages, de cailloux, d'éléments de la nature, et des figures mixtes, visages humains sur fond rustique, avec des corps métamorphosés mêlés à des drapés antiques.

Quatre principales formes de moule sont attestées, toujours plus ou moins incurvées, parfois même semi-cylindriques : la forme dite «sarcophage » suit le contour de la tête et des épaules ; la forme rectangulaire peut correspondre à la partie médiane ou à la partie inférieure du terme ; la forme rectangulaire, avec un large bandeau plat à la base, sert de socle aux termes. Un terme métamorphosé était ainsi constitué de quatre moules. La composition des figures à l'antique est moins certaine. Il existe néanmoins un exemple avec des pieds reposant sur une base (EP 2715), montrant que ce personnage était lui aussi composé de quatre parties - tête et épaules, bras, genoux et pieds -, mais nous n'avons retrouvé dans la collection qu'un unique exemplaire de ce type.

Il existe également des moules de forme triangulaire, plus atypique, que nous avons nommée écoinçon. Ils peuvent appar- 


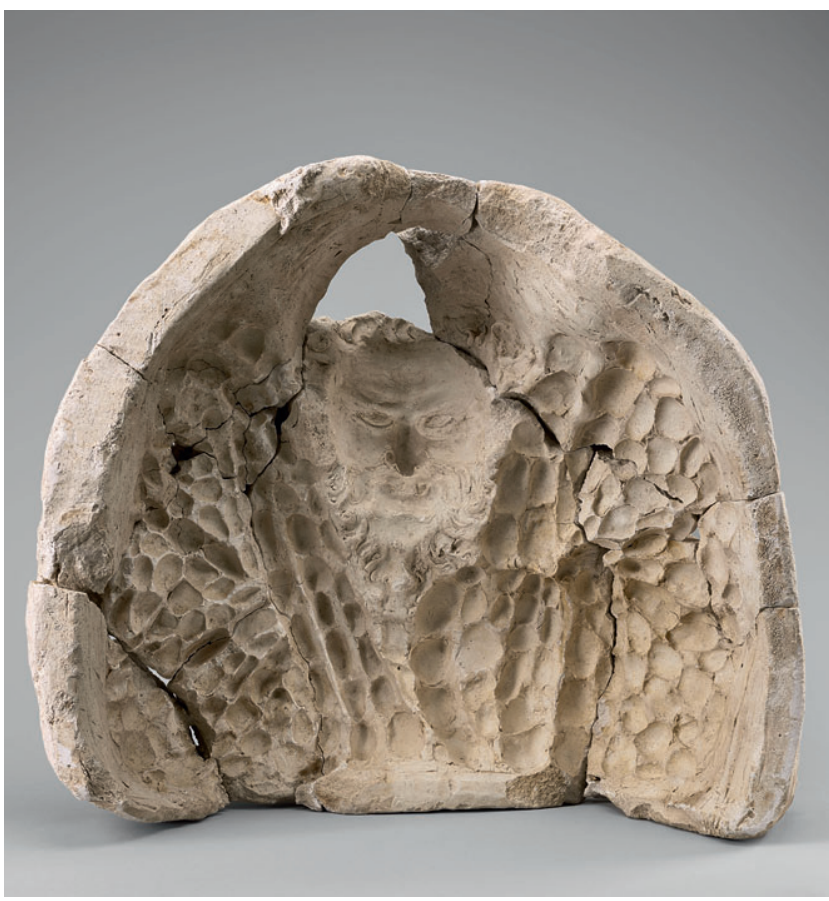

Fig. 2 a. Atelier de Bernard Palissy, Paris, fouilles des Tuileries, troisième quart du Xvi ${ }^{\mathrm{e}}$ siècle, Moule d'un buste d'homme avec empreintes de galets, plâtre (H. $68 \mathrm{~cm}$, L. $62 \mathrm{~cm}$, Pr. $33 \mathrm{~cm})$, Écouen, musée national de la Renaissance, EP 2720. (C) RMN-Grand Palais (musée national de la Renaissance, château d'Écouen)/Mathieu Rabeau.

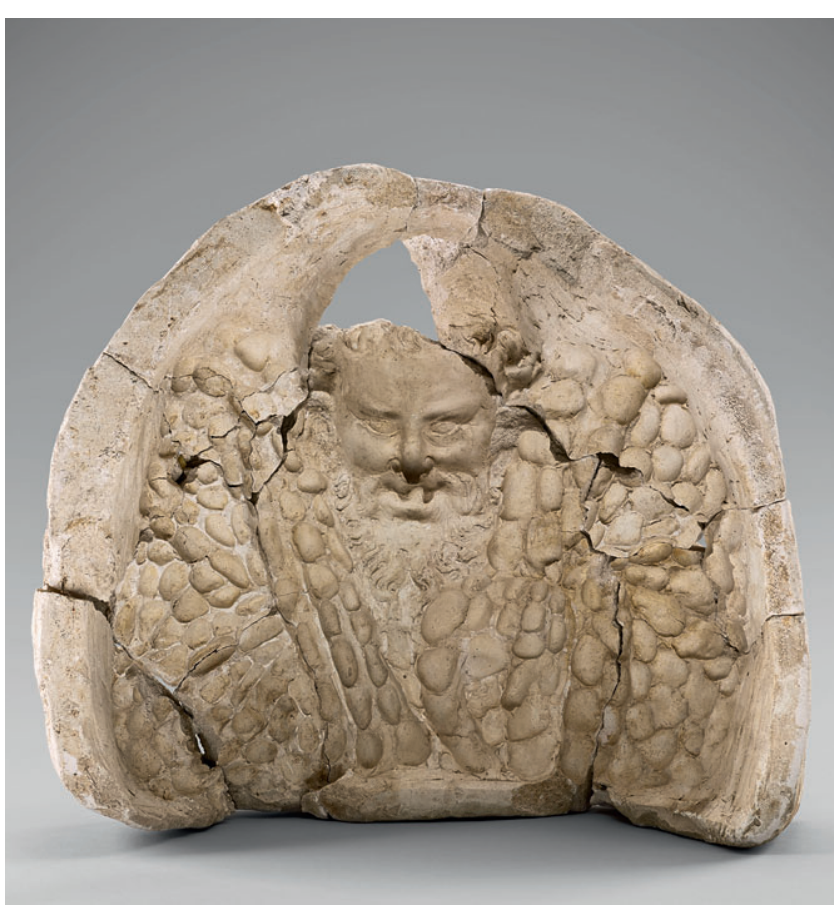

Fig. 2 b. Prise de vue en lumière rasante donnant l'effet en positif du moule 2 a. (C) RMN-Grand Palais (musée national de la Renaissance, château d'Écouen)/Mathieu Rabeau. tenir aux termes, mais également au décor de la grotte, comme le laisseraient penser ceux présentant une main tenant un tissu, sorte de velum suspendu (EP 2719, EP 2739 et EP 2745).

Afin d'évaluer la quantité de personnages présents dans la collection, nous avons débuté nos travaux par l'étude des portraits, termes et antiques. Une quinzaine ont été dénombrés dont trois visages féminins, une dizaine de visages masculins et deux indéterminés, masculin ou féminin, entièrement ornés de « roche brute » pour l'un (EP 2742) et de coquillages et cailloux pour le second (EP 2749).

Dix figures humaines sont mises en scène à l'antique. Parmi elles, deux femmes : l'une vêtue mais dénudant son sein gauche, un diadème ornant sa chevelure, l'autre ne portant ni vêtement ni attribut. Tous les personnages masculins affichent une spécificité dans leur habit : fibule, cuirasse, ceinture, léonté pour l'un d'entre eux (fig. 1 a et b, EP 2717). Sept sont barbus et chevelus, un est imberbe.

Les dimensions des moules d'un même registre sont proches, à l'exception d'un exemple plus large (fig. 2 a et b, EP 2720). Ce dernier met en scène un visage humain, masculin, avec un corps de galets aux formes allongées. Le décor de fond également constitué de galets se confond avec le buste. Les contours du moule sont arrondis et non de type «sarcophage », la largeur en est bien plus importante. Ces dimensions le rendaient imposant et le distinguaient des autres figures.

Les termes présentent un mélange de décors très variés, tirés du répertoire classique des grottes rustiques : coquillages,pierres, roche brute - des bandes de galets alternant parfois avec des bandes de roche brute -, textile, drapé, impressions de végétaux. Plusieurs moules sont ornés d'un décor plus simple, deux uniquement d'empreinte de textile (EP 2740 et EP 3434), et cinq autres de roche brute sur trame de tissu (EP 2697, EP 2699, EP 2702, EP 2742 et un dernier sans numéro d'inventaire).

Dans sa description de la grotte d'Anne de Montmorency, Palissy évoque des termes dont le décor est proche de moules conservés au fort d'Écouen, notamment trois d'entre eux avec coquilles et pierres (EP 2713, EP 2722 - fig. 3 a et b - et EP 2749). Le rapprochement est tentant, mais ne demeure qu'à l'état d'hypothèse.

La transformation naturelle d'un terme, «mangé de l'air (...) rustiqué, \& enrichy de diverses mousses, herbes, \& pierres estranges ${ }^{3}$ ", est une transformation matérielle et profonde, difficile à visualiser à la lecture du négatif du moule. Avec une orientation particulière de la lumière, la photographie permet cependant d'appréhender le positif de certains moules (fig. 2 a et b, fig. 3 a et b).

Les précisions que donne Palissy sur les couleurs des termes démontrent un véritable aboutissement de sa création. Son travail de glaçure visait à accentuer le rendu du vieillissement de la matière. Les moules qui sont des intermédiaires ne nous livrent que des aspects techniques plastiques, avec une lecture complexe et abstraite. Le fragment de terre cuite glaçurée, exemple d'un moulage réalisé à partir d'un grand moule, nous donne un aperçu des intentions de Palissy et de ses recherches sur la glaçure (fig. 4). 


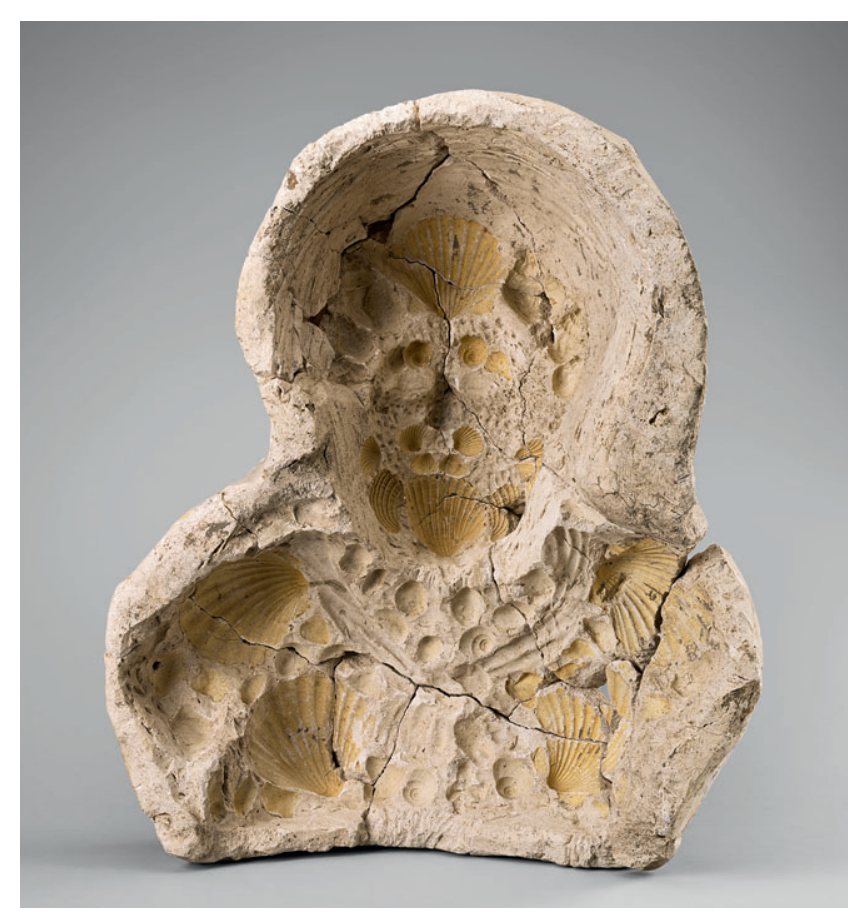

Fig. 3 a. Atelier de Bernard Palissy, Paris, fouilles des Tuileries, troisième quart du $\mathrm{XvI}^{\mathrm{e}}$ siècle, Moule d'un buste d'homme avec empreintes de coquillages, plâtre (H. $66 \mathrm{~cm}, \mathrm{~L} .53,5 \mathrm{~cm}, \operatorname{Pr} .23 \mathrm{~cm}$ ), Écouen, musée national de la Renaissance, EP 2722. @ RMN-Grand Palais (musée national de la Renaissance, château d'Écouen)/Mathieu Rabeau.

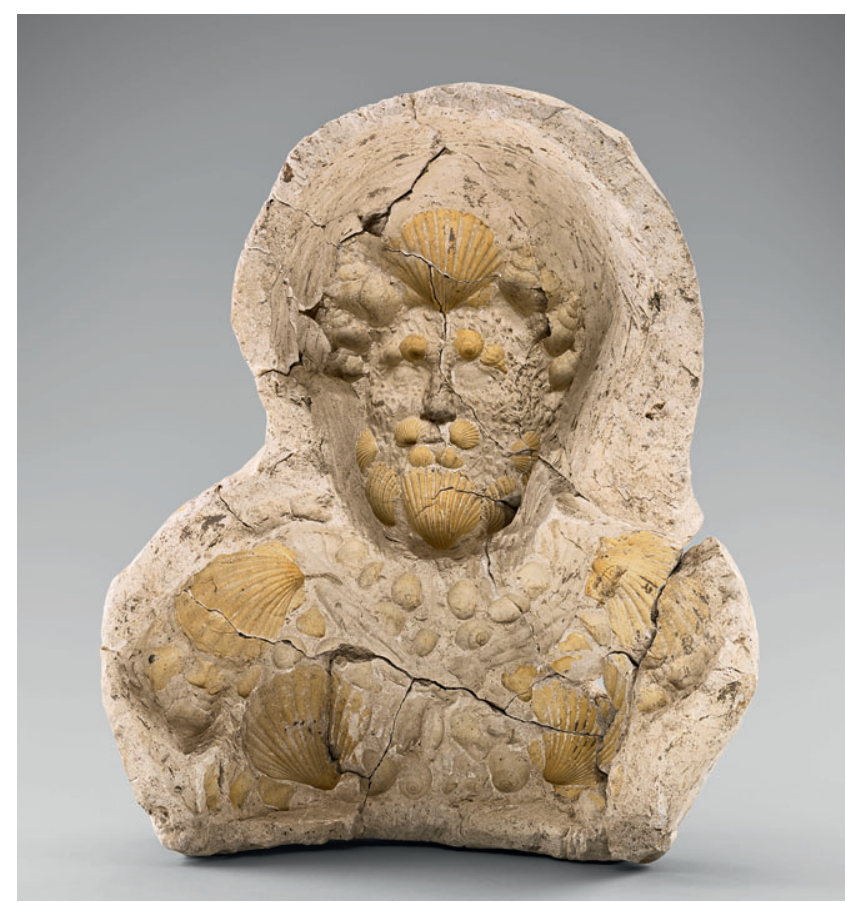

Fig. 3 b. Prise de vue en lumière rasante donnant l'effet en positif du moule 3 a. @ RMN-Grand Palais (musée national de la Renaissance, château d'Écouen)/Mathieu Rabeau.

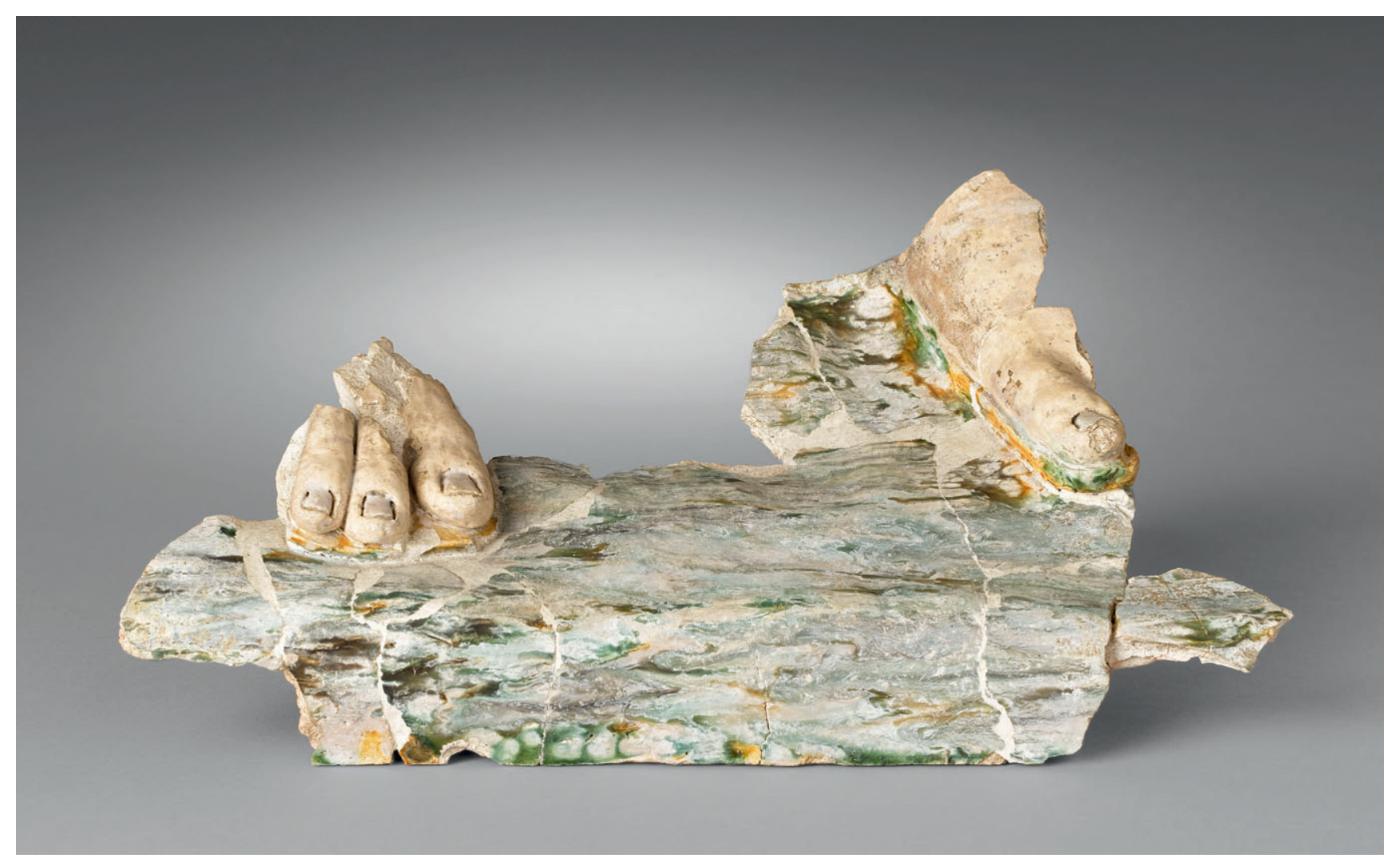

Fig. 4. Atelier de Bernard Palissy, Paris, fouilles des Tuileries, troisième quart du Xvi ${ }^{\mathrm{e}}$ siècle, Fragments de pieds sur support rocheux, terre cuite glaçurée (L. $43 \mathrm{~cm}, 1.18,5$ cm, Pr. 22,5 cm), Écouen, musée national de la Renaissance, EP 464.1.

() RMN-Grand Palais (musée national de la Renaissance, château d'Écouen)/Stéphane Maréchalle. 


\section{Les étapes de réalisation des grands moules}

Les moules sont à creux perdu ${ }^{4}$ : ils sont tous constitués d'un seul tenant, sans aucune pièce ni trace d'assemblage. Pour des facilités de mise en œuvre et de démoulage, l’original devait être nécessairement en terre. Lors de son élimination du moule, il était détruit. De l'œuvre, il ne subsiste plus que son empreinte en négatif au creux du moule.

\section{Le modèle original}

Avant de concevoir ses grands moules, Palissy a certainement réfléchi à plusieurs variantes, pratiqué des essais qui devaient à la fois lui permettre d'imaginer ses compositions et de lever des contraintes techniques. De ces préambules, quelques vestiges nous sont parvenus comme la partie inférieure d'un visage féminin, rare positif en plâtre de cette collection (fig. 5), provenant peut-être du moulage d'une œuvre antique, des assemblages de coquillages sur une matrice composée de mortiers grossiers (fig. 6), représentant des fragments de décors et un visage (fig. 7) et, enfin, de petites plaques de moules de coquillages. Les compositions sur mortier semblent plutôt correspondre à des études et non pas au modèle original moulé, car leur élimination serait particulièrement fastidieuse dans un moule à creux perdu. Ces pièces ne témoignent donc pas de la technique des moules, mais évoquent les recherches de Palissy, ses projets, ses aspirations.

L'élaboration du modèle original, a priori en terre, relève de la combinaison de plusieurs techniques : moulage, modelage, empreinte (textile notamment) et incrustation de décors tels que les coquillages.

Les techniques ne sont pas aisées à reconnaître, les surfaces des moules étant souvent altérées. Le plâtre a parfois perdu son épiderme et les cassures nombreuses rendent difficile, sans collage - ce qui est le cas de la plupart des moules restés à l'état de fragments -, la lecture des volumes. Enfin, d'impressionnantes fissures, formées lors de la prise du plâtre, déforment quelquefois les modelés.

Néanmoins, Palissy a dû recourir au moulage sur nature pour des visages tels que celui de la femme au diadème (EP 2434) dont le profil senestre est déformé - la chaleur dégagée par le plâtre durant sa prise pouvait ramollir les chairs et les déformer ${ }^{5}$ - et celui de l'homme très expressif et ridé (fig. 2 a et b-EP 2720). En revanche, des éléments tels que les yeux, cheveux et barbes étaient modelés.

La prudence s'impose néanmoins au sujet du moulage sur nature pour les visages, car nos observations sous loupe binoculaire n'ont pas permis de déceler les caractéristiques de la peau (ridules, pores, poils), à la différence des tirages en plâtre obtenus à partir des moules découverts lors des fouilles de 1865 à Paris, dont l'empreinte a été prise sur un cadavre (main, torse, jambes) et sur lequel nous avons observé la matière particulière de la peau ${ }^{6}$.

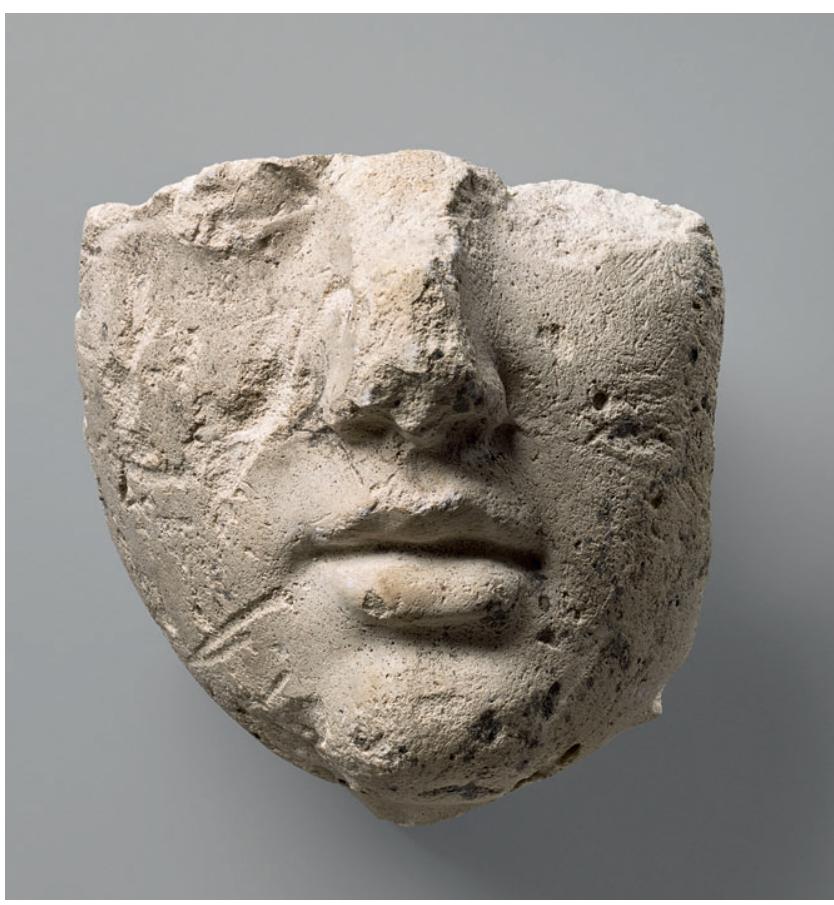

Fig. 5. Atelier de Bernard Palissy, Paris, fouilles des Tuileries, troisième quart du XvI ${ }^{\mathrm{e}}$ siècle, Visage, plâtre $(\mathrm{H} .16 \mathrm{~cm}, \mathrm{~L} .15 \mathrm{~cm}$, Ép. $6 \mathrm{~cm}$ ), Écouen, musée national de la Renaissance, EP 3432. (C) RMN-Grand Palais (musée national de la Renaissance, château d'Écouen)/Mathieu Rabeau.

Des empreintes de toiles de jute, à trame plus ou moins fine, apparaissent souvent sur les figures métamorphosées, soit seules, soit associées aux décors rustiques. Ces marques, régulières et uniformément réparties, correspondent vraisemblablement à une volonté de créer un motif décoratif et non à une technique employée lors du travail de la terre, qui permet d'éviter un séchage trop rapide. Palissy évoque d'ailleurs dans son ouvrage la mise en place de toile imprimée à la surface d'un terme : « les fillez, \& tissure de ladicte toile sont si apparants, qu'il y a peu d'hommes qui ne pensent que ce soit toile naturelle ${ }^{7}$. »

Dans la fabrication de l'original, le positionnement des coquillages et galets pour créer les incrustations du décor constituait la dernière étape avant le moulage.

\section{Le moule}

Une coloration jaune recouvre souvent les empreintes des coquillages. Elle pourrait témoigner de l'utilisation d'agents démoulants, ou encore de l'application d'une barbotine ; elle pourrait aussi provenir du coquillage lui-même. Cette coloration est en cours d'analyses, des prélèvements ont été effectués par le C2RMF sur des pièces non nettoyées.

L'original en terre une fois prêt, Palissy installait un coffrage tout autour de son modèle, probablement élaboré à partir de plaques en terre cuite (rebuts de cuissons ? tuiles ?), 


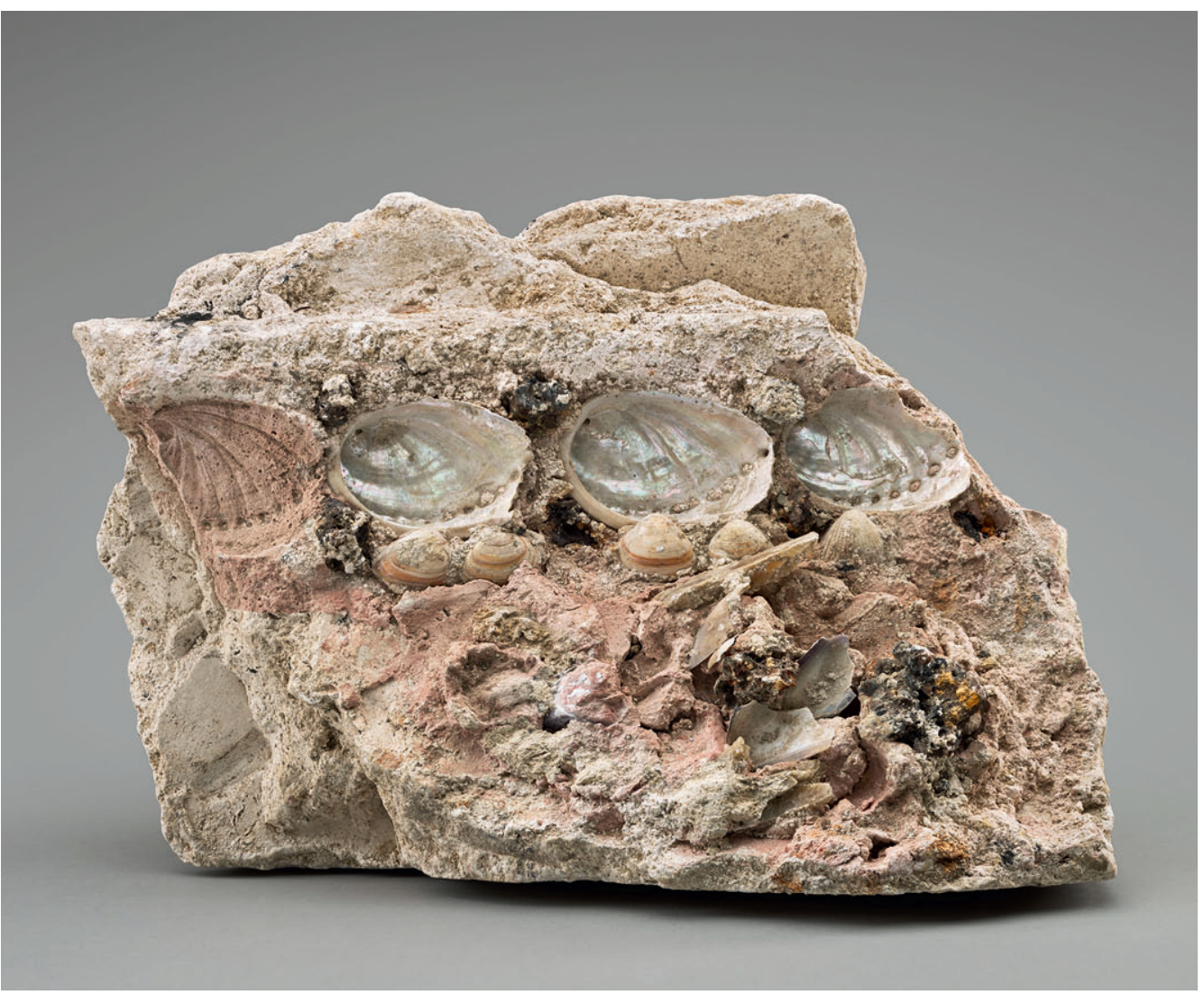

Fig. 6. Atelier de Bernard Palissy, Paris, fouilles des Tuileries, troisième quart du Xvi ${ }^{\mathrm{e}}$ siècle, Décor de rocaille, plâtre et coquilles (H. $30 \mathrm{~cm}$, L. $24 \mathrm{~cm}$, Pr. $19 \mathrm{~cm}$ ), Écouen, musée national de la Renaissance, EP 3433. @ RMN-Grand Palais (musée national de la Renaissance, château d'Écouen)/ Mathieu Rabeau.

ainsi que l'évoquent les marques laissées sur les côtés des moules (plans coupés lisses) ${ }^{8}$. Une première couche de surface était coulée ou peut-être appliquée au pinceau ; des gâchages plus ou moins denses suivaient. Un fond terminait le moule en lui donnant une forme bombée. Pour en améliorer l'adhérence, de larges stries étaient parfois pratiquées avant cette dernière opération. Les coulées successives donnaient une épaisseur importante à l'ensemble : le plâtre d'empreinte est d'environ $1 \mathrm{~cm}$ alors que la coulée du fond peut aller jusqu'à $4 \mathrm{~cm}$.

Les premiers plâtres sont fins ; celui du fond est souvent moins pur et peut contenir des fragments de charbon de bois, des petits cailloux, du sable ou de la brique finement pilée.

Le démoulage s'effectuait avant la prise totale du plâtre pour en faciliter l'opération. Des arrachements sur la face décorée sont souvent visibles. Palissy a dû connaître bien des déceptions à la découverte de ses moules, leur épaisseur ayant en effet souvent provoqué la formation de fissures de prise.

Certains décors, comme celui de la «pierre brute ", étaient mis en place après le démoulage à l'aide de gouge utilisée soit perpendiculairement à la surface, soit de biais, sur un plâtre encore humide.

L'extérieur des moules révèle de nombreuses informations, d'ordre technologique, notamment des inscriptions récurrentes. De grandes lettres majuscules d'environ $10 \mathrm{~cm}$ de hauteur sont gravées avant séchage complet, au revers et sur les côtés de vingt-six moules, de toutes formes. Elles sont parfois incomplètes ou peu lisibles, car elles se situent désormais sur des zones de cassures ${ }^{9}$. Parfois, des croix ont été incisées sur les côtés, nécessairement après démoulage. Leur fonction n'a pas encore été interprétée. Elles pourraient correspondre à la formation d'un personnage/terme ou encore constituer un repère pour leur localisation dans la grotte.

Sept moules ne comportent pas de lettre, sept autres présentent des lettres incomplètes ou illisibles. Les manques et les lettres illisibles compliquent le travail de recomposition des personnages et démultiplient les associations possibles, ce qui accentue le caractère hypothétique de nos propositions.

\section{Le positif: hypothèse ou réalité?}

Une argile grise a été déposée sur la surface des moules. Celle-ci est très différente de la terre d'enfouissement également observée. Ces traces d'argile recouvrent parfois la coloration jaune dans les coquillages et comblent des bulles d'air du plâtre. Il pourrait donc s'agir de résidus de terre d'un tirage ultérieur.

Les prélèvements d'argile grise effectués à l'intérieur et sur les côtés de trois moules (EP 2738, EP 2716 - fig. 8 a et b - et EP 2707) ont été étudiés et analysés par Yvan Coquinot et Nathalie Ganfoldo en 2017 : « Tous les restes d'argiles 


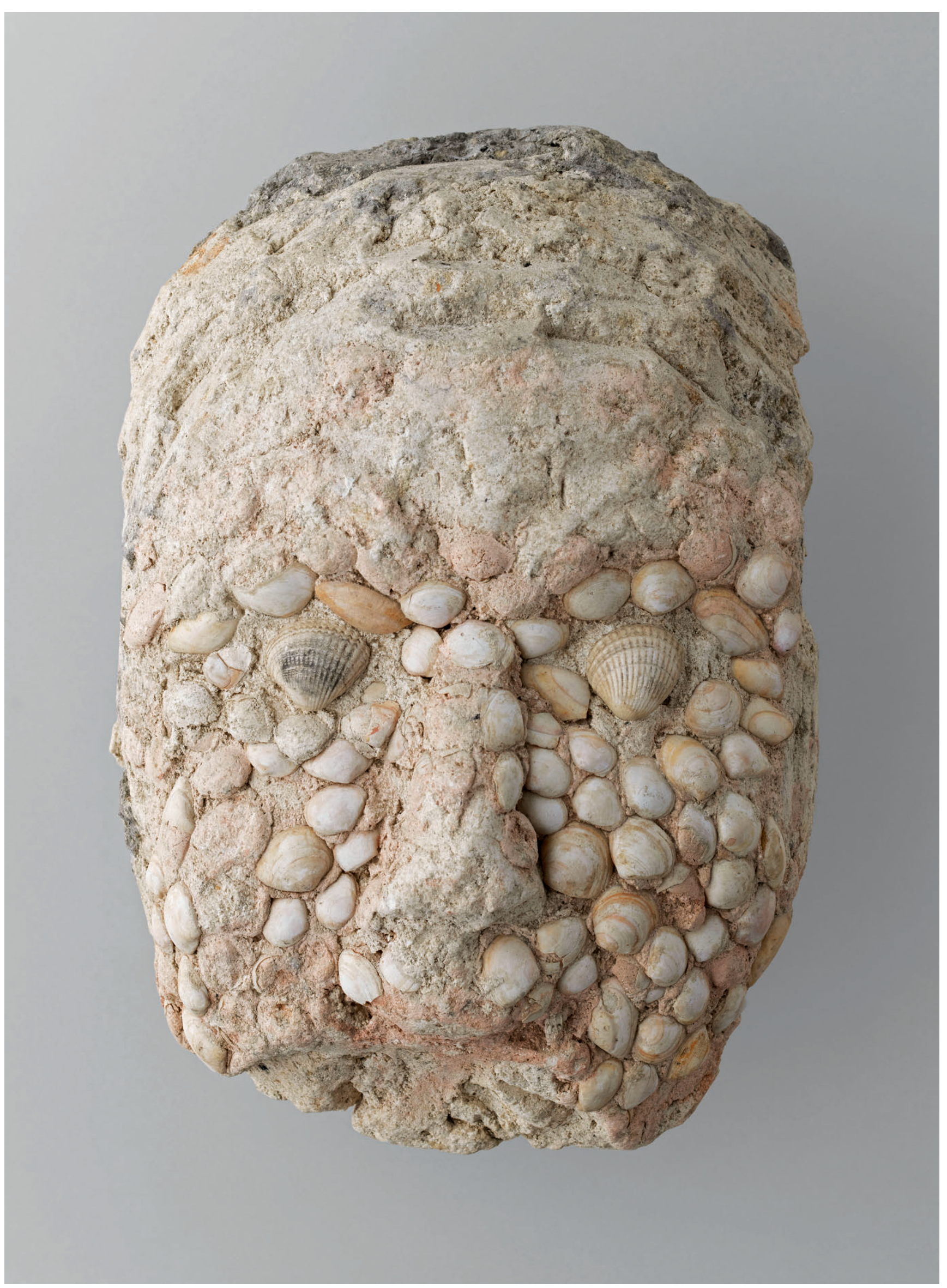


prélevés correspondent à l'argile utilisée par Palissy pour faire de la céramique et non à de la terre du sol. Deux types d'argiles ont pu être distingués, mais elles proviennent toutes d'Île-de-France... La raison de la présence de ces argiles à l'intérieur et à l'extérieur des moules n'apparaît malheureusement pas évidente au vu des résultats de cette étude. Pour l'argile prélevée à l'extérieur des moules (sur le revers), il pourrait s'agir d'une contamination accidentelle dans l'atelier. Pour l'argile retrouvée sur les surfaces ou dans les pores de l'intérieur des moules, il pourrait s'agir également d'une contamination accidentelle dans l'atelier ou de restes des tirages $^{10}$.»

En outre, comment interpréter l'existence de cassures nombreuses et récurrentes ? Résultent-elles de la casse des moules à creux perdu, ce qui signifierait que ceux-ci ont été utilisés et que des tirages ont existé ? Ou bien s'agirait-il d'une destruction volontaire ou encore du simple passage du temps?

La destruction des moules pour récupérer le positif laisse normalement des traces : bûchage, arrachage, traces de décochage, éclats. Nous avons observé sur la plupart des moules des traces de bûchage à peu près au centre du bord supérieur et du bord inférieur, mais pas de coups d'outils à proximité des cassures. L'utilisation des moules pour un tirage demeure donc incertaine.

\section{Les assemblages : quelques exemples}

En 2015, nous avons rédigé pour chaque moule une fiche d'intervention qui comprend la description des archéologues que nous complétons avec des observations techniques de mise en œuvre et un constat d'état de conservation ; nous y ajoutons un résumé de ce qui a été effectué pendant l'étude et des propositions d'interventions.

La rédaction de l'ensemble des fiches a permis l'élaboration d'un tableau synthétique qui facilite la recherche d'assemblage par typologie, lettres, dimensions et nature du plâtre. Par exemple, après recoupements dans cette base de données, nous avons pu établir que la lettre E est la plus fréquente, douze occurrences sur l'ensemble des moules : sur trois moules de type sarcophage, représentant trois portraits masculins à l'antique ${ }^{11}$, sur deux formes rectangulaires décorées de galets et de coquillages ${ }^{12}$ et sur deux bases décorées de galets et de coquillages. À partir de l'assemblage de quatre de ces moules, un terme métamorphosé masculin pourrait être reconstitué : le portrait recouvert de coquillages (EP 2713), avec les deux formes médianes rectangulaires décorées de coquillages et de galets (EP 2698 et EP 2703) et l'une des deux bases (EP 2700 ou EP 2743). La hauteur totale serait de 241 à $244 \mathrm{~cm}$, selon la base utilisée, pour une largeur maximale de $55 \mathrm{~cm}$. Les mesures internes pourraient nous aider à confirmer les assemblages.

D'autres recoupements sont possibles suivant la typologie du décor : plusieurs moules portent par exemple un décor de « roche brute ciselée » associée à une trame de toile de jute.
Le buste EP 2742, les parties médianes EP 2697 et EP 2699 et la base EP 2702 pourraient constituer un autre terme.

La recherche d'assemblage des personnages antiques est plus complexe, le décor ne permettant pas des rapprochements immédiats comme les termes. S'il faut envisager qu'ils se terminent par des pieds, comme le montre un seul moule conservé, on devrait donc en déduire que la plupart sont incomplets, du fait de la disparition de l'original (détruit ou restant à découvrir) - à moins qu'il ne faille imaginer un type de base différent.

Aujourd'hui, des connexions n'ont pas été assurément établies entre les grands moules et les fragments de terre cuite glaçurée, autre champ de recherche qui pourrait donner des éléments de réponse à nos questionnements.

À ce stade de nos travaux, seuls quelques personnages ont été reconstitués. Nous ne pouvons proposer que des hypothèses. L'étude des remontages envisageables est en cours, mais les nombreuses cassures qui empêchent de connaître précisément les dimensions internes des moules et l'état lacunaire de certains d'entre eux constituent autant de freins à cette recherche. En outre, si chaque personnage, terme ou antique, est composé de quatre moules, la collection actuelle, en nombre insuffisant, ne permet pas de tous les reconstituer.

Toutefois, des moules de parties médianes, très incomplets, et des fragments de moules sont encore à étudier. La perspective de les relier entre eux nous permet d'espérer la poursuite des reconstitutions des personnages.

De même, des zones d'ombre demeurent quant à la réalisation des portraits. Les techniques de mise en œuvre et les décors sont homogènes sur l'ensemble de cette collection, cependant le traitement des visages semble comprendre des variantes: moulage d'un visage humain ou bien modelage. Pour achever l'étude des visages, il serait souhaitable de poursuivre les remontages de ces moules, encore à l'état de fragments pour un certain nombre d'entre eux. La compréhension d’un négatif (moule) n'est pas aisée, celle de fragments plus complexe encore. Actuellement, nos études ne permettent pas de préciser si les moules correspondent à une ou deux grottes; espérons que le croisement de toutes ces informations apporte ultérieurement quelques éléments de réponse.

Dernier axe de recherche, l'étude et la restauration des grands moules se poursuit par celles des moules de décors architecturaux, en cours. De nouvelles données compléteront ainsi cette première approche des décors des grottes palisséennes. 


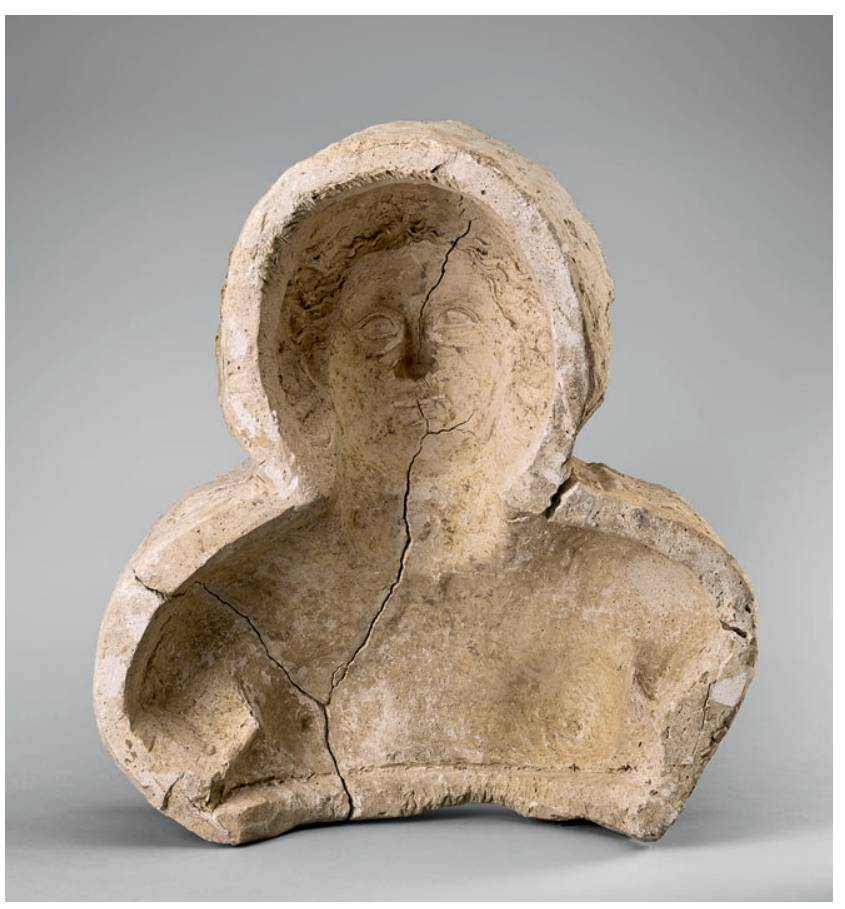

Fig. 8 a. Atelier de Bernard Palissy, Paris, fouilles des Tuileries, troisième quart du $\mathrm{xvI}^{\mathrm{e}}$ siècle, Moule d'un buste de femme vêtue à l'antique, plâtre (H. $58 \mathrm{~cm}, \mathrm{~L} .56 \mathrm{~cm}, \operatorname{Pr} .25 \mathrm{~cm})$, Écouen, musée national de la Renaissance, EP 2716. (C) RMN-Grand Palais (musée national de la Renaissance, château d'Écouen) /Mathieu Rabeau.

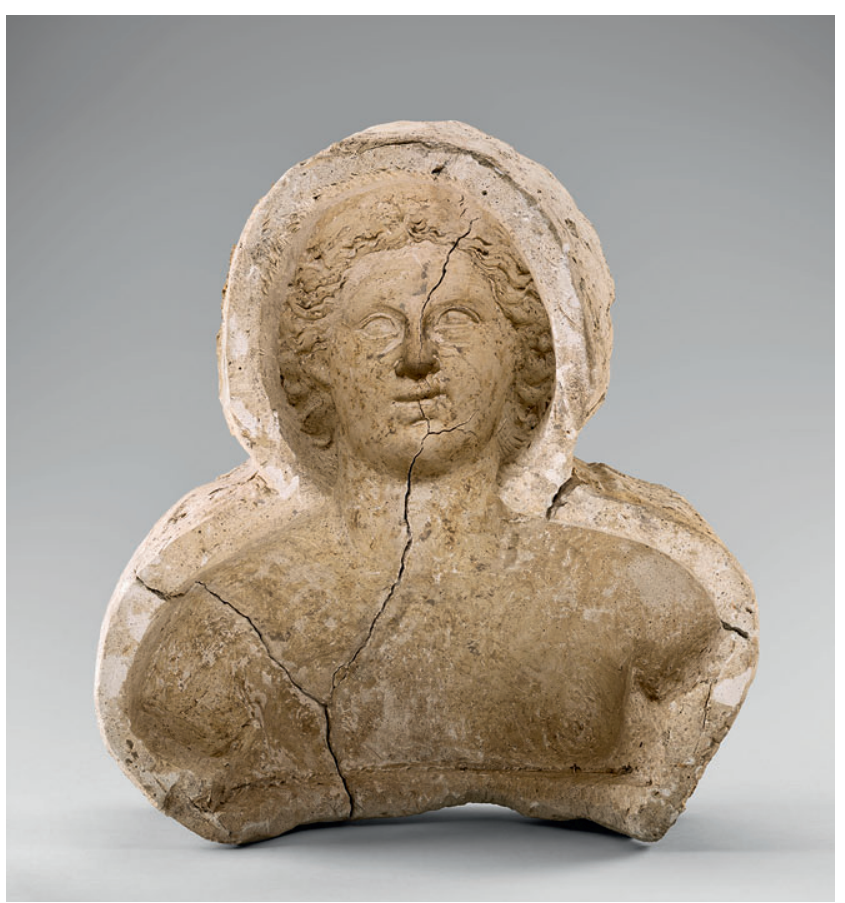

Fig. 8 b. Prise de vue en lumière rasante donnant l'effet en positif du moule 8 a. (C) RMN-Grand Palais (musée national de la Renaissance, château d'Écouen)/Mathieu Rabeau.

\section{Notes}

1. Pour les aspects historiques, voir l'article de A. Gerbier dans ce volume. 2. Dufaÿ, de Kisch, Poulain et al., 1987 , p. 40 .

3. Palissy, 2010, p. 74.

4. Le moule à creux perdu est un moule qui ne donne qu'un seul tirage et que l'on brise lors du dégagement de celui-ci.

5. Lebrun, 1829.

6. Ce tirage (inv. S3615), ainsi que les autres témoignages conservés par le musée Carnavalet sont désormais déposés au musée national de la Renaissance.

7. Palissy, 2010, p. 74.

8. Notre compréhension de la pratique du moulage de Bernard Palissy repose sur un examen des marques sur les côtés, des cassures sur lesquelles apparaît la stratigraphie des différentes coulées et des revers incomplets et/ou altérés qui ont parfois perdu la dernière coulée.

9. Nous avons ainsi relevé les lettres $\mathrm{C}$,

D, E, F, G, H, I, K, L, M, N, T, Z.

10. Coquinot, Gandolfo, 2017, Paris : "Souvent, jusqu'au Xıx ${ }^{\mathrm{e}}$ siècle, le plâtre pouvait parfois contenir des impuretés, notamment des morceaux de charbon, dues au contact d'une partie du gypse avec le combustible lors de la cuisson (en raison du type de four utilisé jusqu'au début du $\mathrm{XIX}^{\mathrm{e}} \mathrm{s}$.). De ce fait, ce plâtre n'était pas de qualité suffisante pour réaliser des moulages ou des enduits d'intérieur. On l'utilisait alors plutôt dans la construction, ou comme Palissy, pour faire des éléments "techniques", avec des grands volumes, et sans intérêt esthétique. "

11. EP 2713, visage avec un décor de coquillages ; EP 2714, visage avec un vêtement drapé à l'antique ; EP 2735, visage barbu avec une cuirasse. 12. EP 2698 et EP 2703.

\section{Bibliographie}

Amico L., 1987, « Les céramiques rustiques authentiques de Bernard Palissy ", Revue de l'Art, n ${ }^{\circ} 78$, p. 61-69.

Amico L., 1996, À la recherche du paradis terrestre. Bernard Palissy et ses continuateurs, Flammarion, Paris.

Audiat L., 1868, Bernard Palissy, étude sur sa vie et ses travaux, Didier et Cie, Paris. Réédition, 2012.

Barthe G., 2002, Le plâtre : l'art et la matière, Créaphis, Ivry-sur-Seine.
Cat. Exp. Paris, 2001, À fleur de peau : le moulage sur nature au XIX $X^{e}$ siècle [Exp. Paris, musée d'Orsay, octobre 2001-janvier 2002], Bajac Q., de FontRéaulx D., Didi-Huberman G., Macel C., Papet E. (com. général.), Schnalke T., Sorel P., RMN Éditions, Paris.

Dufaÿ B., de Kisch Y., Poulain D., Roumégoux Y., Trombetta P. J., 1987, "L'atelier parisien de Bernard Palissy », Revue de l'Art, $\mathrm{n}^{\circ}$ 78, p. 33-60.

Lebrun, 1829, Manuel complet du mouleur ou l'art de mouler en plâtre, carton-pierre, carton-cuir, carton, cire, plomb, argile, bois, écaille, corne, etc., Librairie encyclopédique de Roret, Paris.

Lecoq A. M., 1987, « Morts et résurrections de Bernard Palissy ", Revue de l'Art, $\mathrm{n}^{\circ} 78$, p. 26-32.

Palissy B., 2010, Euvres complètes, Fragonard M.-M. (dir.), seconde édition revue et annotée, Honoré Champion, Paris.

\section{Document inédit}

Coquinot Y., Gandolfo N., 2017, Compte-rendu d'étude provisoire, $\mathrm{C} 2 \mathrm{RMF}$. 